\title{
Newer innovations in interventional endoscopic ultrasound
}

\section{Dongwook Oh, Do Hyun Park, Min Keun Cho, Kwangwoo Nam, Tae Jun Song, Sang Soo Lee, Dong-Wan Seo, Sung Koo Lee, Myung-Hwan Kim}

Gastrointest Endosc 2015 early online

Endoscopic retrograde pancreatography (ERP) and transpapillary drainage are an effective procedure for treating pancreatic ductal obstruction due to various causes. However, it fails in $3-10 \%$ of patients because of various reasons such as inaccessible papilla or duodenal obstruction..$^{[1]}$ In these situations, advent of endoscopic ultrasound (EUS) has made endoscopic drainage possible either by rendezvous procedures or by directly accessing the dilated blocked pancreatic duct from the stomach or duodenum. ${ }^{[2,3]}$ During EUS-guided pancreatic duct drainage (EUS-PD), a stent can be placed both via antegrade new transmural tract and/or papilla using rendezvous techniques. ${ }^{[4]}$ The literature on EUS-PD is limited and most studies have reported the use of plastic stents for antegrade drainage. ${ }^{[4]}$ The plastic stents are associated with increased risks of pancreatic fluid leakage as well as migration and moreover tend to get blocked earlier. Self-expanding metallic stents (SEMS) have larger diameter and have been shown to have prolonged patency in various benign and malignant biliary strictures. Fully covered self-expandable metal stent (FCSEMS) have been previously used for biliary and pancreatic ductal strictures with encouraging results. ${ }^{[5,6]}$ However, FCSEMS have not been previously used for EUS-PD because of concerns about stent migration as well as the risk for cross-stream blockage of main pancreatic duct (MPD) that cover the membrane of the FCSEMS. The authors report their experience with FCSEMS for transmural stenting via EUS-PD in patients in whom ERP had failed or was technically not feasible as well as in whom EUS-guided rendezvous procedures also failed or patients who had painful malignant pancreatic ductal strictures.

The authors conducted a retrospective analysis of prospectively collected database of 25 patients with painful obstructive pancreatitis who underwent EUS-PD after failed ERP. The study was done in two parts: First was a pilot study where temporary FCSEMS was placed in patients with benign MPD obstruction, and this was followed by an observational follow-up where patients were divided into two groups. These groups were permanent FCSEMS placement group $(n=13 ; 52 \%)$ and temporary FCSEMS placement group $(n=12 ; 48 \%)$. The endoscopist decided the allocation of the patient to either temporary or permanent group. Patients with malignant MPD strictures $(n=11)$ or surgically altered anatomy and complete MPD obstruction even if benign $(n=2$; chronic pancreatitis) were considered for allocation to permanent FCSEMS placement group.

A modified FCSEMS of 6 or $8 \mathrm{~mm}$ diameter, $6-10 \mathrm{~cm}$ length, made up of nitinol wire and silicon covered from inside of wire mesh, with both end have anchoring flaps covered with membrane which helps in anti-migration (M.I. Tech, Seoul, South Korea) was used in this study. The size of the pancreatic duct determined the choice of diameter of the FCSEMS (6 or $8 \mathrm{~mm}$ ). All the procedures were done by a single experienced endoscopist. The guide wire was placed in the pancreatic duct after puncturing it with a 19G needle, and the tract was subsequently enlarged sequentially using a triple lumen needle knife and $4 \mathrm{~mm}$ Hurricane balloon (Boston Scientific). Thereafter, FCSEMS was placed in the MPD, and passage of FCSEMS across the fistula tract along with the flow of contrast medium or pancreatic fluids through the enteral side of FCSEMS was considered as technical success. Improvement in symptoms with any reduction of pain was considered as clinical success.

Technical and clinical success were achieved in all 25 patients (100\%) with median procedure time of $25 \mathrm{~min}$. Repuncture and positioning of the EUS fine needle because of poor axis for FCSEMS placement were required in $8 / 25$ patients (32\%). EUS-guided pancreatogastrotomy was the most common approach $(n=23)$ with duodenum and jejunum being used for access in one patient each. Pain scores improved significantly after FCSEMS placement and 50\% (7/14) patients with benign etiology and $36.4 \%$ (4/11 patients) patients with malignant etiology discontinued analgesic medications.

Five patients (25\%) experienced minor adverse events (4 self-limited abdominal pain and 1 minor bleeding). None of the patients had definite or aggravated upstream ductal dilatation by cross-stream blockage of the MPD that covered membrane of FCSEMS on follow-up computed tomography scans, or no patient had stent-induced ductal changes on ERP done at the time of removal of FCSEMS. No stent was migrated during follow-up, and no stent revision was required in intention to permanent stenting group. Five patients died because of cancer progression without stent-related adverse events. The mean stent patency was $126.9 \pm 66.54$ days during the mean follow-up period of $221.1 \pm 190.48$ days.

\section{Commentary}

EUS-PD is a difficult and complex procedure with the requirement of specialized accessories and an experienced endosonologist. It is still done at very few centers because of the complexity of the procedure as well as increased risk 
of various complications such as pancreatic juice leakage, perforation, and migration of stents. Usually, plastic stents have been used for EUS-guided pancreaticogastrostomy or pancreaticoduodenostomy, and SEMS have not been used for this purpose. The authors, of the current study, have demonstrated that FCSEMS can be successfully placed for EUS-PD and has very high both clinical as well as technical success. FCSEMS has several advantages as compared to plastic stents such as longer stent patency rate, lesser migration rates, and fewer repeat interventions. The results of this study have opened a new era in endoscopic pancreatic interventions and have given us a new armamentarium for endoscopic approach in these difficult and tricky clinical situations that usually needed surgery previously.

Single center study with the procedure being performed by a single experienced interventionist who has tremendous experience in EUS-PD, small sample size and short-term follow-up are some of the important limitations of this study. In spite of these limitations, this study is taken us a step ahead in therapeutic EUS and opened a new door of minimally invasive endoscopic intervention for patients with dilated MPD and failed transpapillary drainage, a group of patients that earlier required surgery. The results of this study are exciting, but further comparative multicentric studies evaluating the long-term safety of these stents are needed before they are routinely used in clinical practice.

\section{Forward-viewing versus oblique-viewing echoendoscopes in the diagnosis of upper gastrointestinal subepithelial lesions with endoscopic ultrasound-guided fine needle aspiration: A prospective, randomized, crossover study}

\section{Ippei Matsuzaki, Ryoji Miyahara, Yoshiki Hirooka, Kohei Funasaka, Eizaburo Ohno, Masanao Nakamura, Hiroki Kawashima, Akiko Nukaga, Yoshie Shimoyama, Hidemi Goto}

\section{Gastrointest Endosc 2015; 82:287-95}

The subepithelial lesions in upper gastrointestinal (GI) tract are now being increasingly detected due to advancement in endoscopic techniques and wide availability of endoscopes. There are various radiological imaging methods to characterize the nature of the subepithelial lesions, but endoscopic ultrasound (EUS), due to its ability to correctly identify the layer of origin and ability of tissue sampling, has surpassed all imaging techniques for proper evaluation of subepithelial lesions. ${ }^{[7,8]}$ The diagnostic accuracy of EUS-guided fine needle aspiration (EUS-FNA) in upper GI subepithelial lesion varies from $70 \%$ to $90 \%{ }^{[9,10]}$ The oblique-viewing echoendoscope (OV-EUS) is the standard equipment for EUS-FNA due to its wide scanning range and elevator function for easy maneuverability for FNA needles. However, lack of a forward endoscopic view and difficulty in fixing the target are important limitations of OV-EUS. The forward-viewing echoendoscope (FV-EUS) is a new development in the field of interventional EUS and because of its forward view and relative ease to fix the target, it is presumed to have better ease in performing FNA, and few studies have indeed shown encouraging results. ${ }^{[1-13]}$ However, lack of elevator and narrow scanning range are important limitations of FV-EUS scope. The experience with FV-EUS in evaluating GI subepithelial lesions is limited, and therefore authors in this study first time compared the OV-EUS and FV-EUS for its diagnostic accuracy with EUS-FNA in upper GI subepithelial lesions.

Authors conducted a prospective, randomized, cross-over trial enrolling 41 patients from a pool of 78 patients (median age 64 years) with upper GI subepithelial lesions. Echoendoscope, used in this study, was the newly developed FV-EUS (GIF-Y0007- UCT or TGF-UC260J; Olympus) with the attachment of a transparent hood (D-201-16403; Olympus) to the tip for fixing the lesion and OV-EUS (GF-UCT240AL-5; Olympus). All patients underwent EUS-FNA procedures with both the echoendoscopes with the echoendoscope order being selected randomly using computer-generated numbers. The needle used for the FNA purposes was disposable 19G needle, and a $22 \mathrm{G}$ or $25 \mathrm{G}$ needle was used when there was failure by $19 \mathrm{G}$ needle, or there was highly vascularized lesion (EZ Shot2: NA-220H-8019, NA-220H-8022, NA-220H-8025; Olympus). Maximum of 3 passes was taken in each echoendoscopy, and an on-site cytopathologist was not present. The results were compared for diagnostic yield, diagnostic accuracy, tissue sample area, technical efficacy, and adverse events.

All the lesions were endoscopically within the lumen of GI tract and the most common site of tumor location was in the stomach (32 cases) and the most common layer of origin was muscularis propria (38 cases). The overall rate of histological diagnosis did not differ significantly using FV-EUS or OV-EUS ( $80.5 \%$ vs. $73.2 \%$, respectively; $P=0.453)$. The diagnostic yield did not differ using FV-EUS first (21 patients) or OV-EUS first (20 patients). The diagnostic accuracy was $77.2 \%$ and $72.7 \%$ for FV-EUS and OV-EUS, respectively $(P=1.000)$. The significant difference was noted in the tissue sample area, which was 2.46 times more in FV-EUS $(P=0.46)$, and procedure time was significantly lower by FV-EUS (21 min vs. $27 \mathrm{~min} ; P=0.009$ ). There was no significant difference in puncture success rate or a number of passes during EUS-FNA between the two echoendoscopes. A puncture could not be performed in 2 cases by FV-EUS and in 3 cases by OV-EUS. The median number of passes required by both the echoendoscopes was 2 . Four lesions were indeterminate which were diagnosed by repeat EUS-FNA, or bite-on-bite forceps technique, or laparoscopic surgery. One patient had infectious adverse event that was treated by 
broad-spectrum antibiotics. The authors concluded that the diagnostic yields for GI subepithelial lesions are comparable between FV and OV-EUS, but FV-EUS samples a large tissue area in lesser procedure time as compared to OV-EUS scopes.

\section{Commentary}

Until now, OV-EUS is the standard echoendoscope for therapeutic procedure, and FV-EUS scope is a new kid on the block of interventional EUS. The current study is first of its kind which compared FV-EUS and OV-EUS for EUS-FNA of GI subepithelial lesions. The results of the study have not supported the presumed advantage of FV-EUS as the diagnostic yield was found to be comparable between the two scopes. However, the tissue sample area was significantly larger in FV-EUS and procedures took significantly less time in FV-EUS. There are presumed advantages of FV-EUS is the lesions located in fornix, or the head of pancreas, or lesions that are associated with strictures where there is difficulty in performing FNA with OV-EUS scope as these are easily punctured in the straight line, and the puncture site is readily visible on FV-EUS scope. ${ }^{[1]}$ Transparent hood attached to FV-EUS had expanded its capability in fixing the smaller target lesion. Authors have recommended that FV-EUS and 19G needle are reasonable combination for adequate tissue acquisition. FV-EUS may be especially useful in sampling small subepithelial lesions as they are difficult to sample with OV EUS scope. This study had few limitations such as small sample size and lack of definitive diagnosis in a large number of cases. In spite of these limitations, this study has established the similar diagnostic yield and adverse events profile of FV-EUS for EUS-FNA as compared to OV-EUS. Ability to sample larger area and shorter procedure time may make FV-EUS scope, the scope of choice for sampling subepithelial lesions, especially the small ones but further comparative multicentric studies are needed to confirm these results.

\section{Deepak Gunjan, Surinder Singh Rana}

Department of Gastroenterology, Post Graduate Institute of Medical Education and Research, Chandigarh, India

Address for correspondence: Dr. Surinder Singh Rana, Department of Gastroenterology, Post Graduate Institute of Medical Education and Research, Sector 12, Chandigarh - 160 012, India. E-mail: drsurinderrana@yahoo.co.in

\section{References}

1. Widmer J, Sharaiha RZ, Kahaleh M. Endoscopic ultrasonography-guided drainage of the pancreatic duct. Gastrointest Endosc Clin N Am 2013;23:847-61.
2. Sharma V, Rana SS, Bhasin DK. Endoscopic ultrasound guided interventional procedures. World J Gastrointest Endosc 2015;7:628-42.

3. Varadarajulu S, Rana SS, Bhasin DK. Endoscopic therapy for pancreatic duct leaks and disruptions. Gastrointest Endosc Clin N Am 2013;23:863-92.

4. Itoi T, Kasuya K, Sofuni A, Itokawa F, Kurihara T, Yasuda I, et al. Endoscopic ultrasonography-guided pancreatic duct access: Techniques and literature review of pancreatography, transmural drainage and rendezvous techniques. Dig Endosc 2013;25:241-52.

5. Devière J, Nageshwar Reddy D, Püspök A, Ponchon T, Bruno MJ, Bourke MJ, et al. Successful management of benign biliary strictures with fully covered self-expanding metal stents. Gastroenterology 2014;147:385-95.

6. Moon SH, Kim MH, Park do H, Song TJ, Eum J, Lee SS, et al. Modified fully covered self-expandable metal stents with antimigration features for benign pancreatic-duct strictures in advanced chronic pancreatitis, with a focus on the safety profile and reducing migration. Gastrointest Endosc 2010;72:86-91.

7. Rana SS, Sharma R, Sharma V, Bhasin DK. An unusual cause of gastric submucosal bulge on endoscopy. Endosc Ultrasound 2014;3:198-9.

8. Rana SS, Bhasin DK, Rao C, Gupta R. Intramural pseudocysts of the upper gastrointestinal tract. Endosc Ultrasound 2013;2:194-8.

9. Akahoshi K, Oya M, Koga T, Koga H, Motomura Y, Kubokawa M, et al. Clinical usefulness of endoscopic ultrasound-guided fine needle aspiration for gastric subepithelial lesions smaller than $2 \mathrm{~cm}$. J Gastrointestin Liver Dis 2014;23:405-12.

10. Ando N, Goto H, Niwa Y, Hirooka Y, Ohmiya N, Nagasaka T, et al. The diagnosis of GI stromal tumors with EUS-guided fine needle aspiration with immunohistochemical analysis. Gastrointest Endosc 2002;55:37-43.

11. Kida M, Araki M, Miyazawa S, Ikeda H, Kikuchi H, Watanabe M, et al. Fine needle aspiration using forward-viewing endoscopic ultrasonography. Endoscopy 2011;43:796-801.

12. Larghi A, Fuccio L, Chiarello G, Attili F, Vanella G, Paliani GB, et al. Fine-needle tissue acquisition from subepithelial lesions using a forward-viewing linear echoendoscope. Endoscopy 2014;46:39-45.

13. Iwashita T, Nakai Y, Lee JG, Park do H, Muthusamy VR, Chang KJ. Newly-developed, forward-viewing echoendoscope: A comparative pilot study to the standard echoendoscope in the imaging of abdominal organs and feasibility of endoscopic ultrasound-guided interventions. J Gastroenterol Hepatol 2012;27:362-7.

This is an open access article distributed under the terms of the Creative Commons Attribution-NonCommercial-ShareAlike 3.0 License, which allows others to remix, tweak, and build upon the work non-commercially, as long as the author is credited and the new creations are licensed under the identical terms.

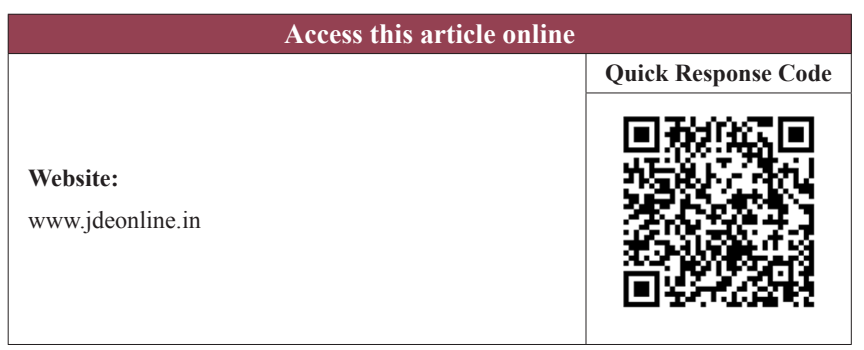

How to cite this article: Gunjan D, Rana SS. Newer innovations in interventional endoscopic ultrasound. J Dig Endosc 2015;6:185-7. 